Supporting Information

\title{
Magnetite/Polymer Brush Nanocomposites with Switchable Uptake Behavior Toward Methylene Blue
}

\author{
Asghar Dolatkhah and Lee D. Wilson* \\ Department of Chemistry, University of Saskatchewan, 110 Science Place, \\ Saskatoon, S7N 5C9, SK, Canada. \\ *E-mail: lee.wilson@usask.ca, Fax: +1-306-966-4730; Tel: +1-306-966-2961
}




\section{Supporting information}

\section{Materials and methods}

\subsection{Materials}

Ferrous chloride (purity $\geq 99.0 \%$ ), ferric chloride (purity $\geq 99.0 \%$ ), acrylic acid (AA, 99\% purity), itaconic acid (IA, purity $\geq 99.0 \%$ ), chitosan powder with $75-85 \%$ deacetylation (CHI, 190 $\mathrm{kDa}$ based on viscosity), ammonium persulfate (APS, 98\% purity), methanol (HPLC grade), and semi-permeable dialysis tubing was obtained from Sigma-Aldrich Canada Ltd. Methylene blue was provided by Alfa Aesar, Canada. All materials were used without further purification and Milli-Q ultrapure water (18.2 M $\Omega . \mathrm{cm})$ was used for the preparation of aqueous solutions herein.

\subsection{Synthesis of PIA and PAA grafted chitosan brushes (PAAgCHI and PIAgCHI)}

The poly (itaconic acid) (PIA) and poly (acrylic acid) (PAA) grafted chitosan polymer brushes were prepared by the method described by Edgefield et al ${ }^{1}$ with slight modification. Briefly, a solution of $1 \% \mathrm{CHI}$ in aqueous acetic acid (1\%; w/v) solution was prepared by dissolving powdered chitosan overnight with stirring in a three neck round-bottom flask equipped with a rubber septum. The solution was purged with nitrogen for $10 \mathrm{~min}$. followed by the addition of APS solution (1\% w/v) to the chitosan solution with stirring for $20 \mathrm{~min}$. A solution of monomer ( $5 \%$ w/v IA or AA) was added to the chitosan solution (as above) dropwise over a 20 min interval under constant nitrogen gas flow. The resulting concentrations were $2.5 \%$ and $0.5 \%$ of monomer and initiator, respectively. The mixture was heated at $60^{\circ} \mathrm{C}$ for $12 \mathrm{~h}$ where the resulting clear solution was cooled and neutralized to $\mathrm{pH} 7$ using $1 \mathrm{M} \mathrm{NaOH}$ solution 
to yield chitosan grafted materials as a precipitate. The product was washed with millipore water several times and dried in a vacuum oven. The PAA grafted chitosan materials were completely soluble in water and the polymer brushes were precipitated in methanol twice to remove side products and low molecular weight unreacted AA. The precipitate was dissolved in millipore water and was dialyzed with water $(3 \times 2 \mathrm{~L}$ changes $)$ for $72 \mathrm{~h}$ to remove unreacted APS. The final product obtained by removal of water with drying in a vacuum oven at $60{ }^{\circ} \mathrm{C}$.

\subsection{Synthesis of MNPs, $\mathrm{Fe}_{3} \mathrm{O}_{4} / \mathrm{PAAgCHI}$ and $\mathrm{Fe}_{3} \mathrm{O}_{4} / \mathrm{PAAgCHI}$ nanohybrids.}

In a typical procedure, $13 \mathrm{~g} \mathrm{FeCl}_{3}(0.05 \mathrm{~mol})$ and $5 \mathrm{~g} \mathrm{FeCl}_{2} \cdot 4 \mathrm{H}_{2} \mathrm{O}(0.025 \mathrm{~mol})$ were dissolved in $200 \mathrm{~mL}$ Millipore water in a three neck round-bottom flask. The reaction was done at $80{ }^{\circ} \mathrm{C}$ in an oil bath under a nitrogen atmosphere. After $10 \mathrm{~min}, 100 \mathrm{~mL}$ of $\mathrm{NaOH}$ solution was added dropwise over $20 \mathrm{~min}$. The solution rapidly turned black, indicating the formation of MNPs. The reaction was stirred for $40 \mathrm{~min}$ at $80{ }^{\circ} \mathrm{C}$ after which the product was collected and purified by five washing cycles with water and magnetic separation to yield magnetic nanoparticles $\left(\mathrm{Fe}_{3} \mathrm{O}_{4}\right)$. To prepare polymer brush/magnetic particles, an aqueous sol of ultrafine MNPs was prepared by sonicating $1 \mathrm{~g}$ of MNPs in $100 \mathrm{~mL}$ Millipore water for $30 \mathrm{~min}$. Then an aqueous solution of PAAgCHI at $\mathrm{pH} 5$ was added drop-wise under $\mathrm{N}_{2}$ gas. PIAgCHI brush was solubilized in $1 \%$ acetic acid overnight and then filtrated to obtain clear PIAgCHI solution. The mixture was sonicated for $1 \mathrm{~h}$ at $70{ }^{\circ} \mathrm{C}$. The product was washed with millipore water and separated magnetically with a permanent magnet to yield PAAgCHI and PIAgCHI modified MNPs as $\mathrm{Fe}_{3} \mathrm{O}_{4} / \mathrm{PAAgCHI}$ and $\mathrm{Fe}_{3} \mathrm{O}_{4} / \mathrm{PIAgCHI}$ nanohybrids. 


\subsection{Characterization}

\subsubsection{IR Spectroscopy}

FT-IR spectra of samples were obtained with a Bio-RAD FTS-40 spectrophotometer where samples were analyzed in reflectance mode. All of the samples were prepared by mixing materials (about $6 \mathrm{mg}$ ) with pure spectroscopic grade $\mathrm{KBr}(60 \mathrm{mg})$ in a mortar and pestle. The DRIFT (Diffuse Reflectance Infrared Fourier Transform) IR spectra were obtained with a resolution of $4 \mathrm{~cm}^{-1}$ and scanning in the range of $400-4500 \mathrm{~cm}^{-1}$. Multiple scans were acquired in reflectance mode and all spectra presented here were baseline corrected relative to spectroscopic grade $\mathrm{KBr}$.

\subsubsection{NMR Spectroscopy}

${ }^{1} \mathrm{H}$ NMR spectra of various samples were recorded at $500 \mathrm{MHz}$ on Bruker Advance 600 or $500 \mathrm{MHz}$ spectrometers at room temperature with tetramethylsilane (TMS) as the internal standard and using either deuterated water or $1 \% \mathrm{HCl} / \mathrm{D}_{2} \mathrm{O}$ as the solvent system. NMR spectra were acquired using standard solvent suppression.

\subsubsection{Thermal Analysis}

Thermogravimetric analysis (TGA) was performed with a TA Instruments (TGA Q500). Samples were placed in an aluminum pan and heated at a scanning rate of $5^{\circ} \mathrm{C} / \mathrm{min}$ under a nitrogen flow $(90 \mathrm{~mL} / \mathrm{min})$. 


\subsubsection{Powder X-ray Diffraction (PXRD)}

X-ray powder diffraction (XRD) patterns of the magnetic products were obtained on a PANalytical Empyrean diffractometer using monochromatic Co-K $\mathrm{K}_{1}$ radiation $(1.79 \AA)$ with a $2 \theta$ scan rate of $3.2^{\circ} \mathrm{min}^{-1}$ from $14^{\circ}$ to $80^{\circ}(2 \theta)$.

\subsubsection{TEM Microscopy}

Transmission electron microscope (TEM) images of samples were obtained using a Hitachi HT-7700 microscope at voltage $100 \mathrm{kV}$. Samples were prepared by depositing a drop of colloidal suspension in ethanol onto a carbon-coated copper TEM grid.

\subsubsection{Dynamic Light Scattering}

The $\mathrm{pH}$-induced sizes of the nanocomposites were performed on a dynamic light scattering spectrometer from X Protein solutions (DynaPro) equipment. The measurements were performed at $20^{\circ} \mathrm{C}$ and a $90^{\circ}$ scattering angle. 
Table S1. Pseudo-First-Order (PFO) and Pseudo-Second-Order (PSO) Adsorption Kinetic Models $(c f$. Eqns $(1)-(6))$.

Pseudo-First-Order

(PFO) kinetics :

$\frac{d C}{d t}=-k_{1} C$ or $C_{t}=C_{o} e^{-k_{1} t}$

$\frac{d q}{d t}=k_{1}\left(q_{e}-q_{t}\right)$ or $q_{t}=q_{e}\left(1-e^{-k_{1} t}\right)$

$\ln \left(q_{e}-q_{t}\right)=\ln q_{e}-k_{1} t$

Pseudo-Second-Order

where $\mathrm{k}_{1}\left(\mathrm{~min}^{-1}\right)=$ the PFO rate constant

$q_{e}=$ the amounts of dye adsorbed at equilibrium

$q_{i}=$ the amounts of dye adsorbed at a given time

(PSO) kinetics:

$$
\begin{aligned}
\frac{d C}{d t} & =-k_{2} C^{2} \text { or } \frac{d q}{d t}=k_{2}\left(q_{e}-q_{t}\right)^{2} \\
\frac{t}{q_{t}} & =\frac{1}{k_{2} q_{e}{ }^{2}}+\frac{t}{q_{e}} \\
q_{t} & =\frac{q_{e}^{2} k_{2} t}{1+k_{2} q_{e} t}
\end{aligned}
$$

where $\mathrm{k}_{2}\left(\mathrm{~g} \mathrm{mg}^{-1} \mathrm{~min}^{-1}\right)$ is the PSO rate constant. 
Table S2. Summary of TGA results of polymer brushes and the corresponding MNCs.

\begin{tabular}{|c|c|c|c|c|c|c|c|}
\hline \multirow[t]{2}{*}{ Sample } & \multicolumn{2}{|c|}{ First weight loss } & \multicolumn{2}{|c|}{ Second weight loss } & \multicolumn{2}{|c|}{ Third weight loss } & \multirow[t]{2}{*}{ Total weight loss (\%) } \\
\hline & $\begin{array}{l}\text { Derivative } \\
\text { peak }\left({ }^{\circ} \mathrm{C}\right)\end{array}$ & $\begin{array}{l}\text { Weight } \\
\text { loss (\%) }\end{array}$ & $\begin{array}{l}\text { Derivative } \\
\text { peak }\left({ }^{\circ} \mathrm{C}\right)\end{array}$ & $\begin{array}{l}\text { Weight } \\
\text { loss (\%) }\end{array}$ & $\begin{array}{c}\text { Derivative } \\
\text { peak }\left({ }^{\circ} \mathrm{C}\right)\end{array}$ & $\begin{array}{l}\text { Weight } \\
\text { loss (\%) }\end{array}$ & \\
\hline PIAgCHI & 79 & 5.6 & 253 & overlap & 309 & 51.6 & 57.2 \\
\hline $\mathrm{Fe}_{3} \mathrm{O}_{4} / \mathrm{PIAgCHI}$ & 55 & 1.4 & 267 & 5.2 & - & - & 6.6 \\
\hline $\mathrm{Fe}_{3} \mathrm{O}_{4} / \mathrm{AAgCHI}$ & 70 & 2.5 & 259 & 5.5 & 422 & 4.6 & 12.7 \\
\hline
\end{tabular}


Table S3. Literature Comparison of Adsorption Capacities of Various Adsorbent Materials Toward MB in Aqueous Solution.

\begin{tabular}{|c|c|c|c|}
\hline Adsorbent & $\begin{array}{l}\text { Adsorption } \\
\text { capacity } \\
(\mathbf{m g} / \mathrm{g})\end{array}$ & Limitations and Comments & Reference \\
\hline $\begin{array}{l}\text { Carbon nanotube } \\
\text { ponytails (CNPs) }\end{array}$ & 150 & $\begin{array}{l}\text { Facile regenertion/High cost/Low adsorption capacity } \\
\text { / lack of magnetic property }\end{array}$ & 2 \\
\hline Graphene oxide & 389.8 & $\begin{array}{l}\text { good adsorption capacity/fascile seperation/ lack of } \\
\text { magnetic properties }\end{array}$ & 3 \\
\hline $\begin{array}{l}\text { Poly(L-dopa)-Based } \\
\text { Polyelectrolyte Complexes }\end{array}$ & 467.1 & $\begin{array}{l}\text { Good adsorption capacity/lack of magneti } \\
\text { property/High cost and expensive } \\
\text { renewable materials }\end{array}$ & 4 \\
\hline $\begin{array}{l}\text { humic acid-modified perlite } \\
\text { (HA/EP) }\end{array}$ & 82.8 & $\begin{array}{l}\text { Low cost /low adsortpion capacity/dificult to separate } \\
\text { and regerenration }\end{array}$ & 5 \\
\hline Carbon nanotubes (CNTs) & 35.4 & $\begin{array}{l}\text { low adsortpion capacity/dificult to separate and } \\
\text { regerenration }\end{array}$ & 6 \\
\hline Graphene & 153.9 & $\begin{array}{l}\text { Easy preparation/low adsortpion capacity/dificult to } \\
\text { separate and regerenration }\end{array}$ & 7 \\
\hline PANI nano particles & 85.4 & $\begin{array}{l}\text { Fascile synthesis and low cost/low adsortpion } \\
\text { capacity/dificult to separate and regerenration }\end{array}$ & 8 \\
\hline PANI/ $\alpha-Z r P$ Nanocomposites & 156.3 & $\begin{array}{l}\text { Low cost/fascile synthesis/relatively low adsorption } \\
\text { capacity/lack of magnetic property and difficult to } \\
\text { regeneration }\end{array}$ & 9 \\
\hline Multiwalled Carbon & 399 & $\begin{array}{l}\text { Good sorption capacity/High cost lack of magnetic } \\
\text { properties }\end{array}$ & 10 \\
\hline $\begin{array}{l}\text { (EDTAD)-modified magnetic } \\
\text { chitosan (EMC) }\end{array}$ & 113.3 & Low sorption capacity & 11 \\
\hline $\begin{array}{l}\text { Polyacrylamide Grafted } \\
\text { Xanthan Gum/nanocilica }\end{array}$ & 497.5 & $\begin{array}{l}\text { Good sorption capacity/low cost/lack of magnetic } \\
\text { property/difficult regeneration }\end{array}$ & 12 \\
\hline Polyaniline hydrogel & 71.2 & $\begin{array}{l}\text { low cost/Low sorption capacity/lack of magnetic } \\
\text { properties }\end{array}$ & 13 \\
\hline Iron sludge & 99.4 & magnetic properties/low cost/ Low sorption capacity & 14 \\
\hline Magnetic chitosan composite & 45.1 & magnetic properties/low cost/Low sorption capacity & 15 \\
\hline $\begin{array}{l}\text { Magnetite/polymer brush } \\
\text { nanocomposites }\end{array}$ & 470.2 & $\begin{array}{l}\text { High adsorption capacity/facile and green } \\
\text { synthesis/low cost/easy recoverability/magnetic } \\
\text { properties/pH-responsive }\end{array}$ & This study \\
\hline
\end{tabular}




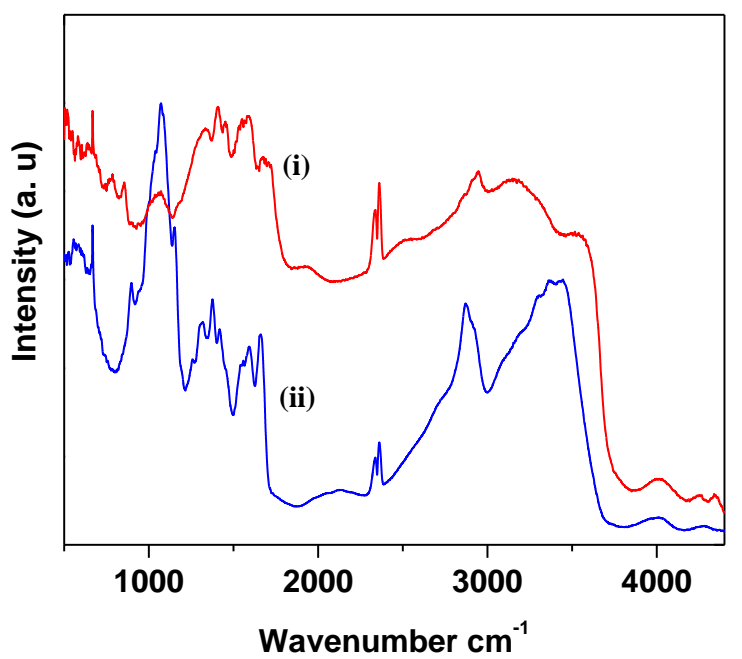

(a)

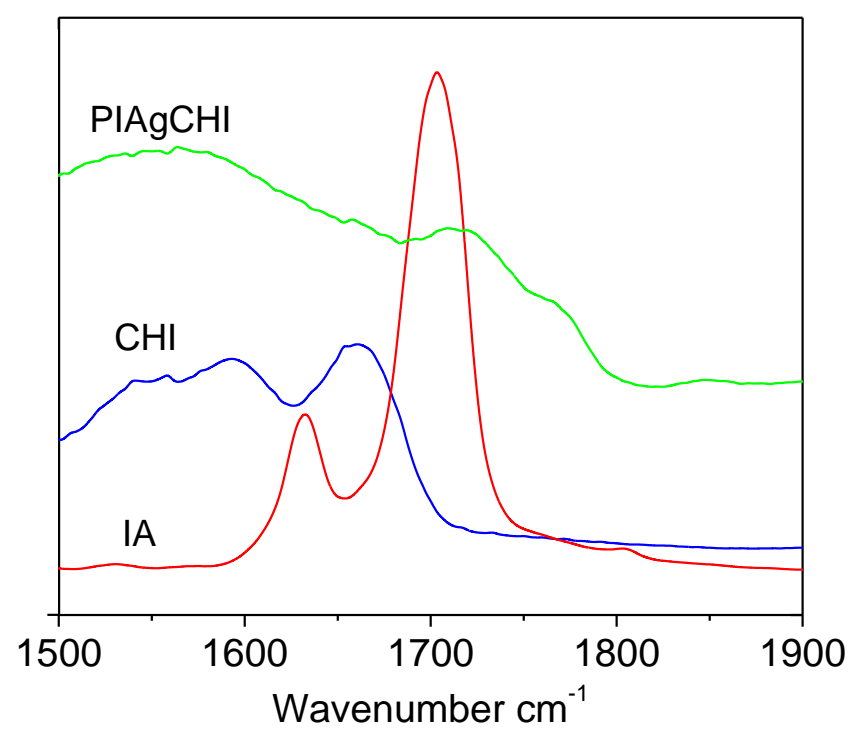

(b) 


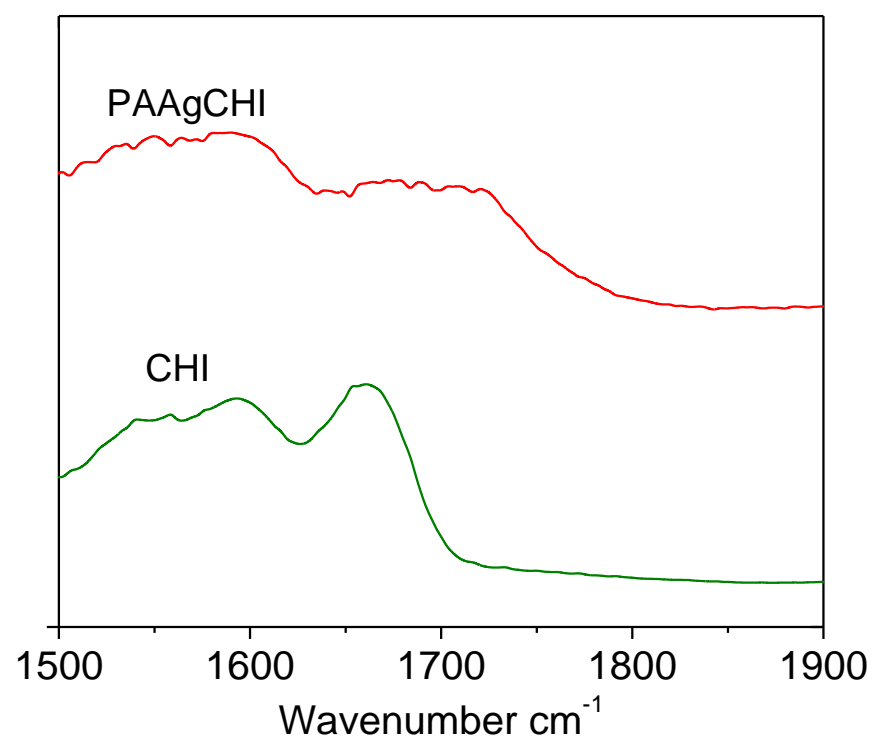

(c)

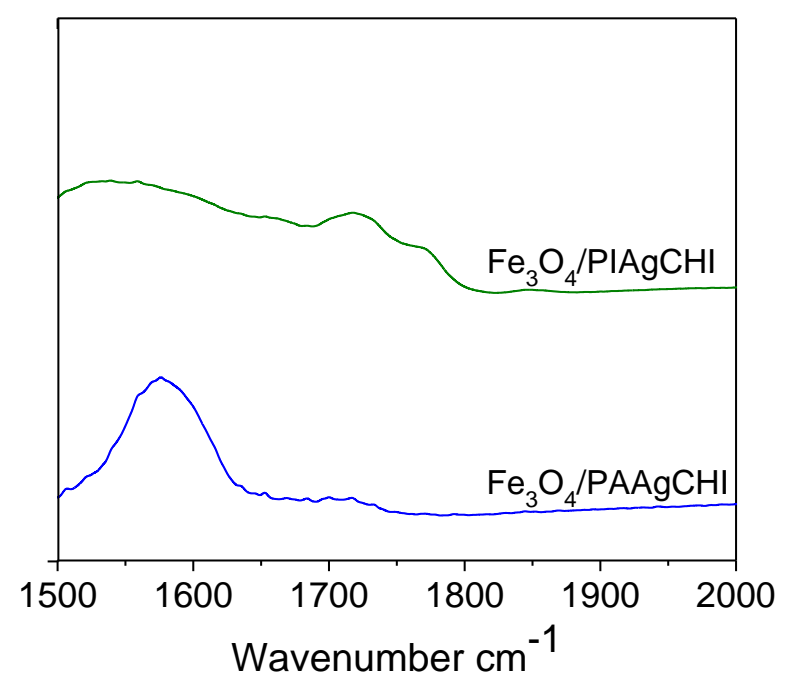

(d) 


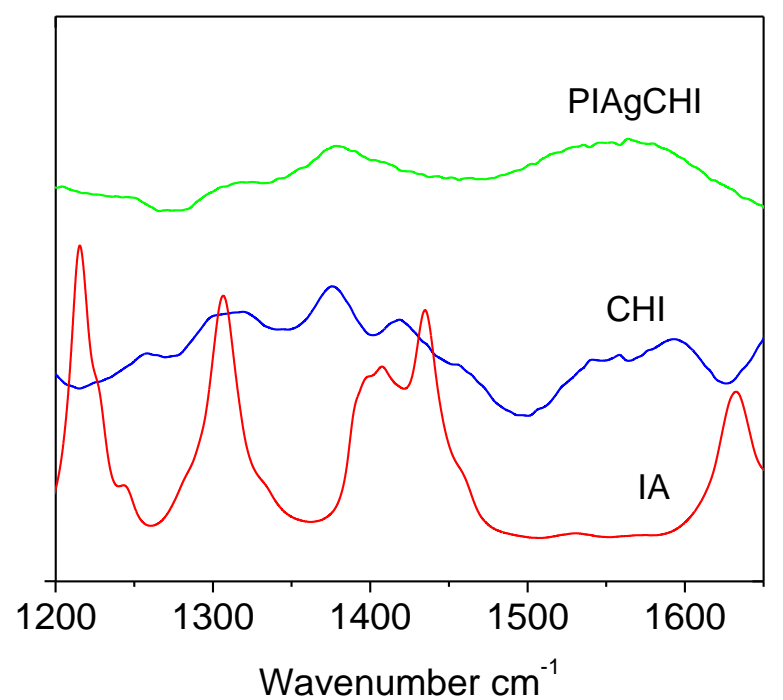

(e)

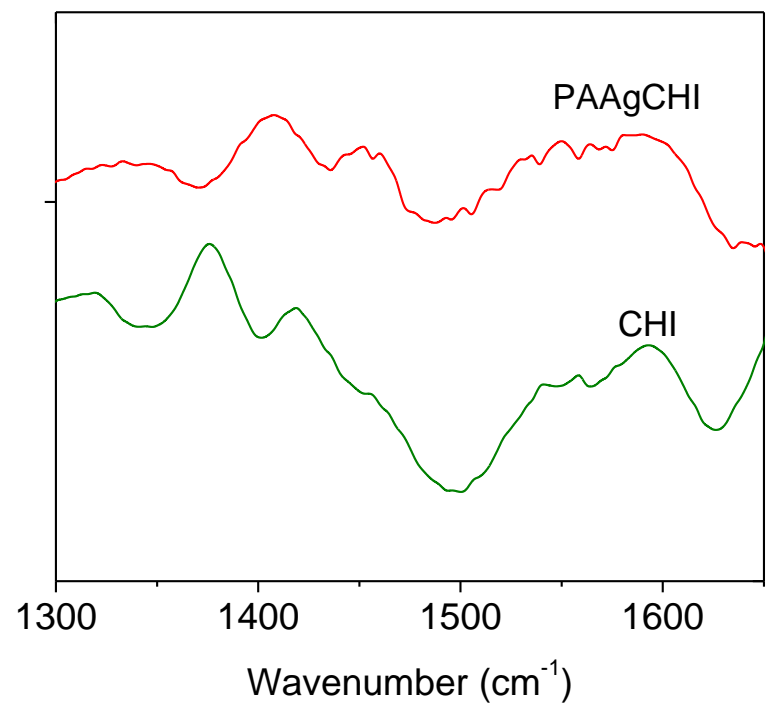

(f) 


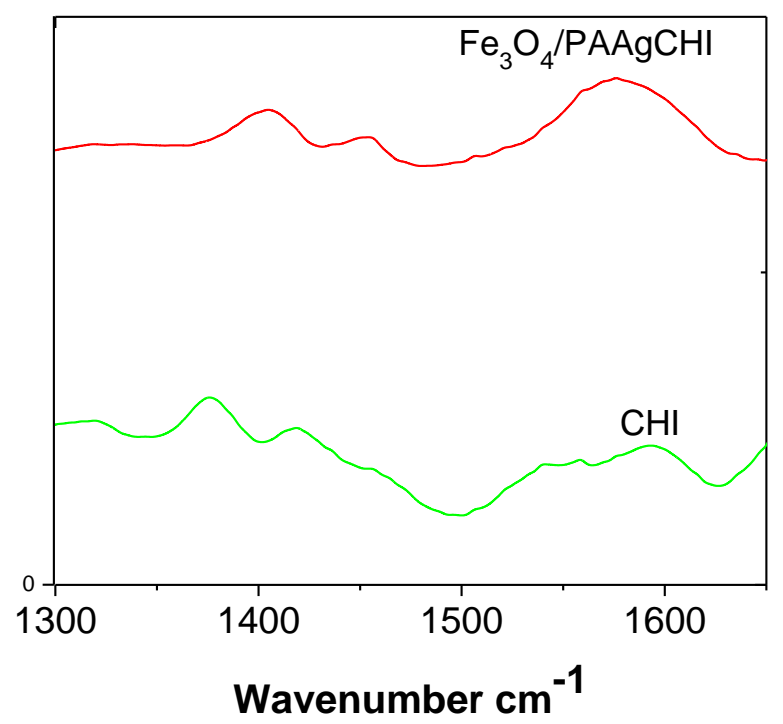

(g)

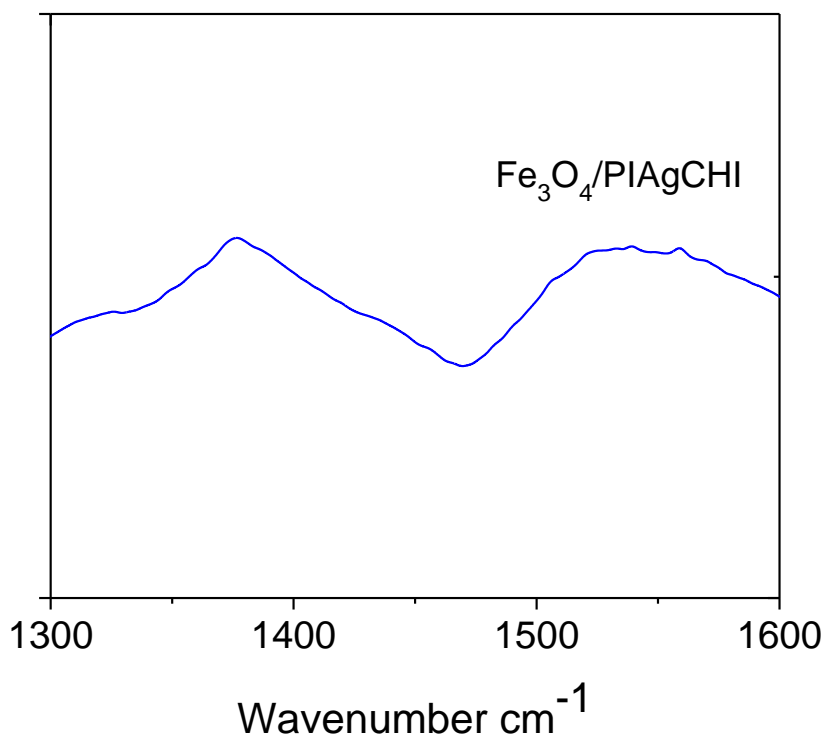

(h)

Figure S1. FT-IR spectra of the PAAgCHI polymer brush (i) and pristine chitosan (ii) (a), CHI, PAAgCHI, PIAgCHI, $\mathrm{Fe}_{3} \mathrm{O}_{4} / \mathrm{PIAgCHI}$ and $\mathrm{Fe}_{3} \mathrm{O}_{4} / \mathrm{PAAgCHI}$ in the range of carbonyl groups $(\mathrm{b}, \mathrm{c}, \mathrm{d})$ and symmetric/asymmetric streching vibrations for $\mathrm{COO}^{-}$groups $(\mathrm{e}, \mathrm{f}, \mathrm{g}, \mathrm{h})$. 


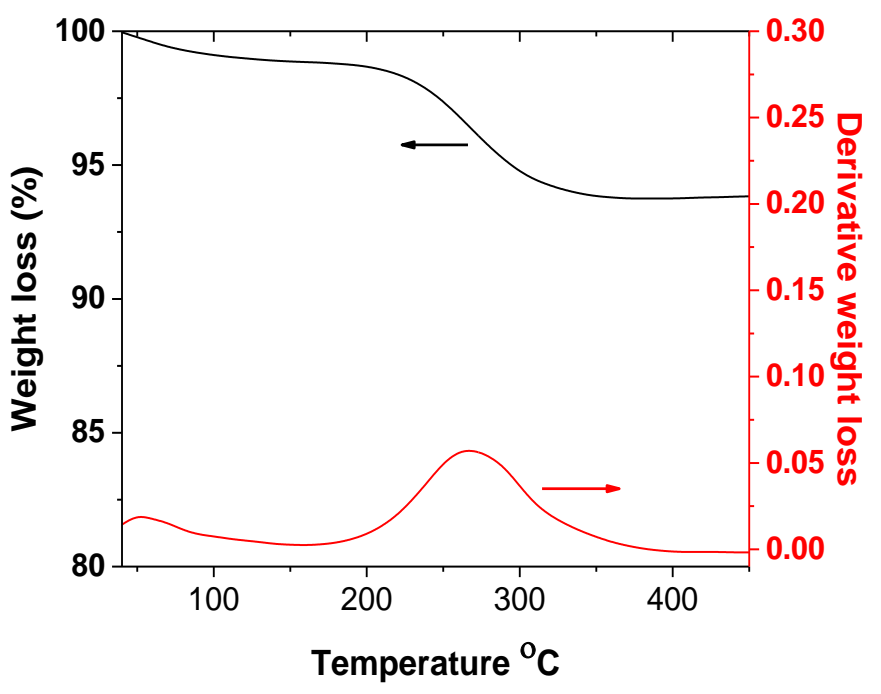

Figure S2. TGA and derivative thermal gravimetry (DTG) profile of $\mathrm{Fe}_{3} \mathrm{O}_{4} / \mathrm{PIAgCHI}$ assemblies. 


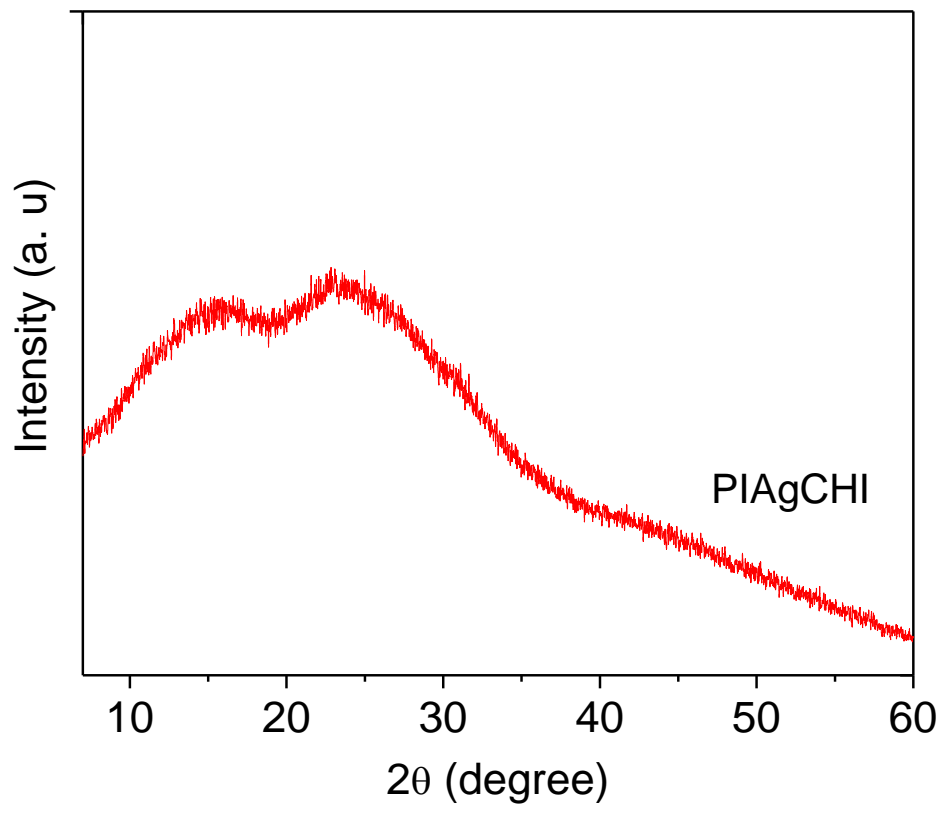

(a)

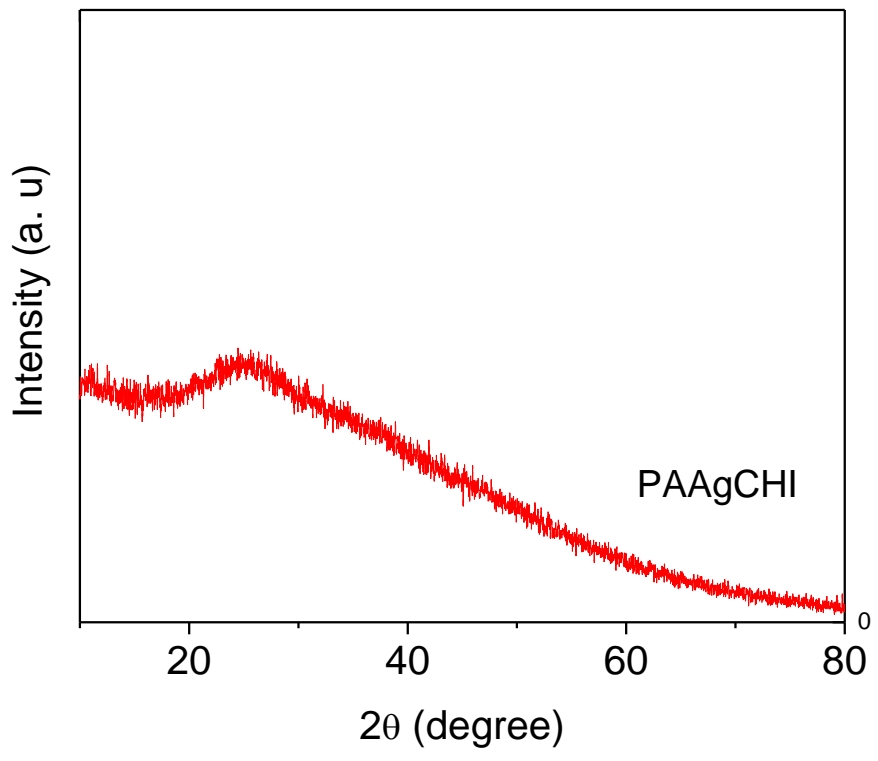

(b)

Figure S3. PXRD patterns for PIAgCHI (a), and PAAgCHI (b) brushes. 


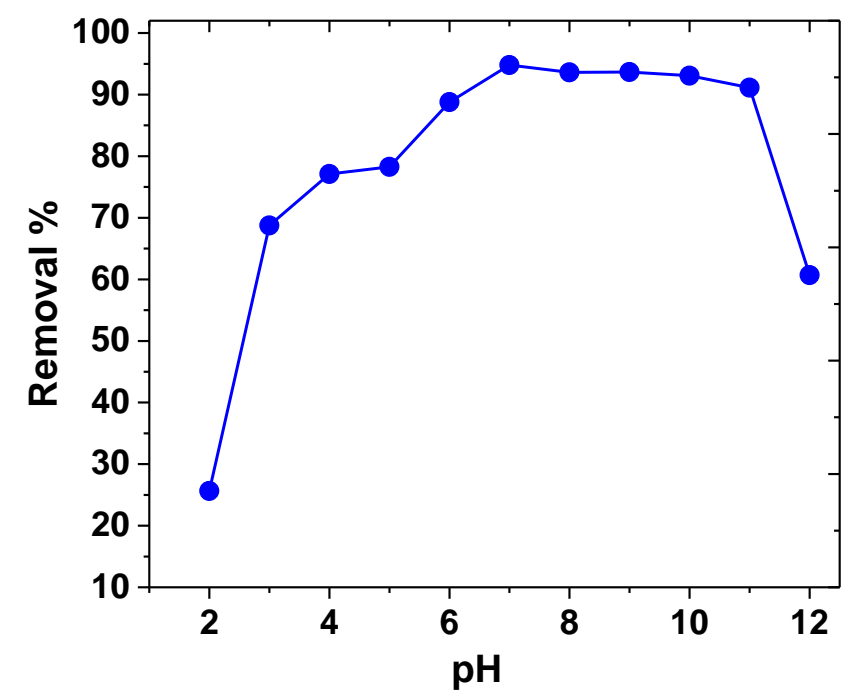

(a)

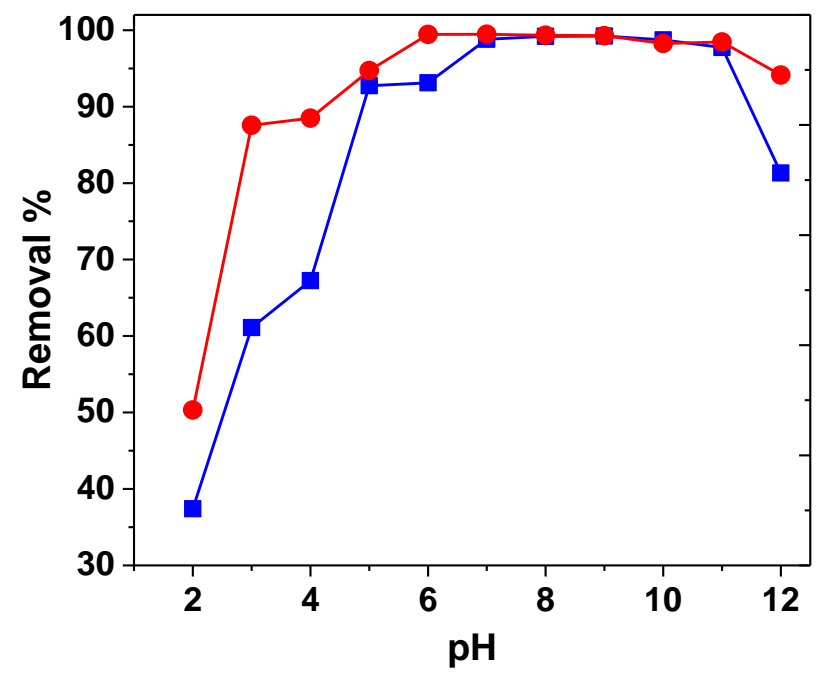

(b)

Figure S4. Effect of solution $\mathrm{pH}$ on $\mathrm{MB}$ adsorption at $298 \mathrm{~K}$ : (a) PIAgCHI; (b) $\mathrm{Fe}_{3} \mathrm{O}_{4} / \mathrm{PIAgCHI}(\bullet)$ and $\mathrm{Fe}_{3} \mathrm{O}_{4} / \mathrm{PAAgCHI}(\boldsymbol{\bullet})$. Experimental conditions: graph (a): $\mathrm{m}=$ $10 \mathrm{mg}, \mathrm{t}=20 \mathrm{~min}, \mathrm{C}_{0}(\mathrm{MB})=0.01 \mathrm{mM}, \mathrm{V}=3 \mathrm{ml}$. graph $(\mathrm{b}): \mathrm{m}=10 \mathrm{mg}, \mathrm{t}=20 \mathrm{~min}, \mathrm{C}_{0}$ $(\mathrm{MB})=0.06 \mathrm{mM}, \mathrm{V}=3 \mathrm{ml}$. 


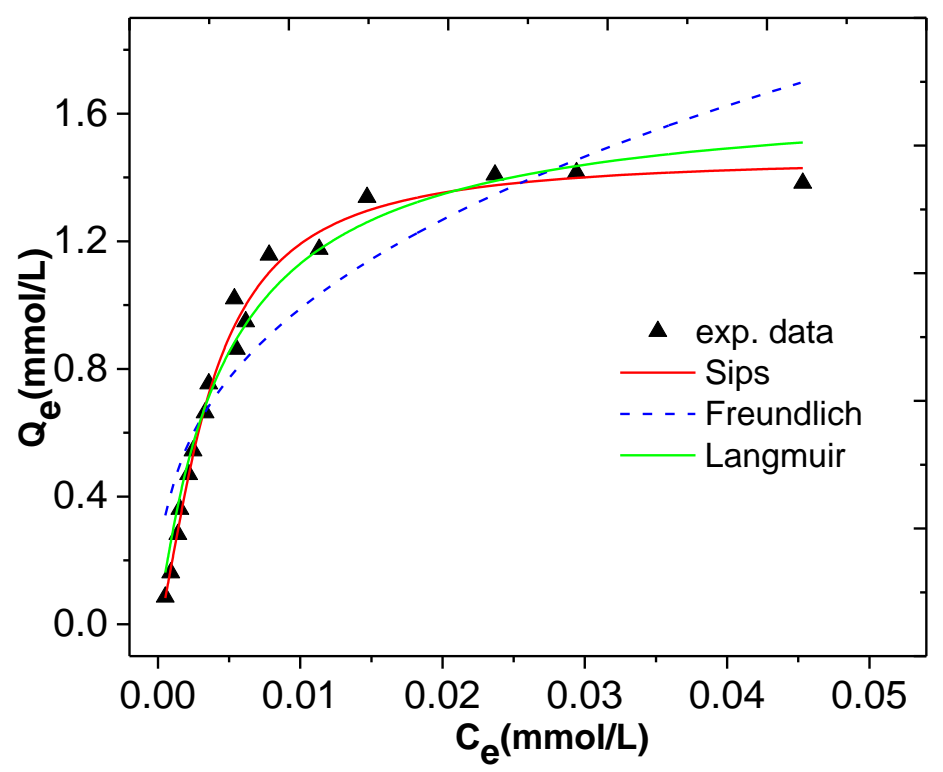

Figure S5. Isotherm sorption results for the $\mathrm{Fe}_{3} \mathrm{O}_{4} / \mathrm{PIAgCHI}$ composite system with $\mathrm{MB}$. 


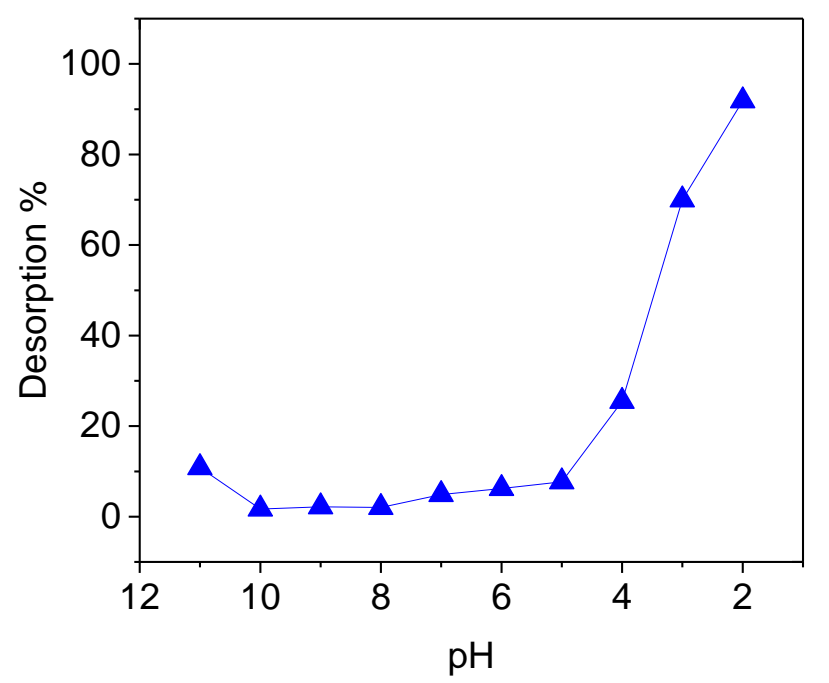

(a)

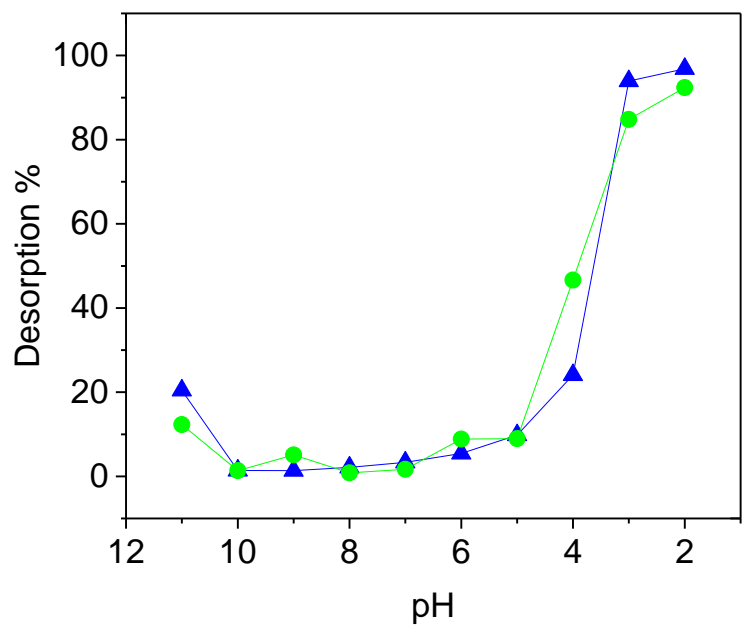

(b)

Figure S6. Dependence of MB desorption from PIAgCHI brush ( $\boldsymbol{\Delta}$ ) and PIAgCHI polymer brush containing nano hybrids (•) (a) and PAAgCHI polymer brush nano hybrid at variable $\mathrm{pH}$ conditions $(\boldsymbol{\Delta})(\mathrm{b})$. 


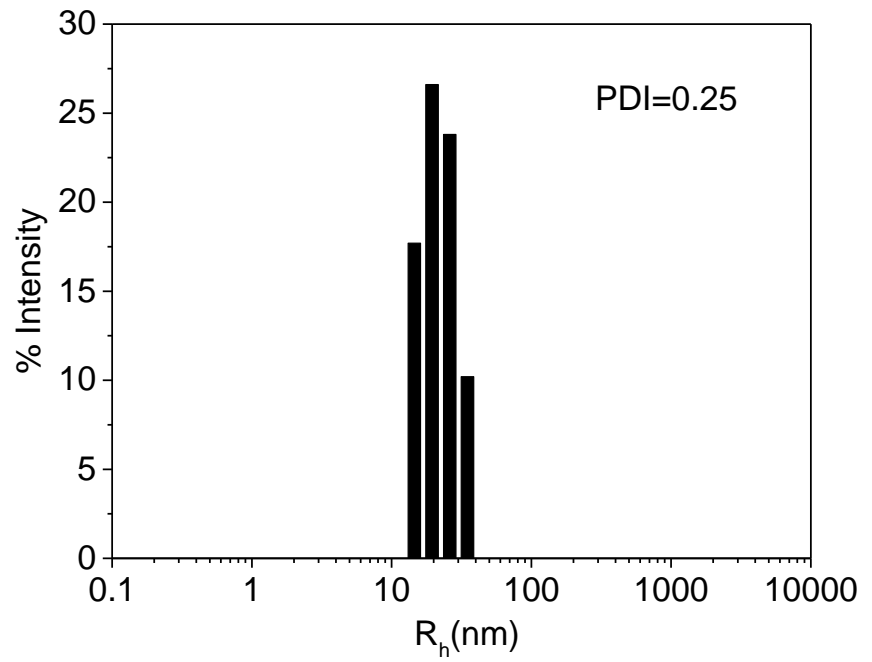

(a)

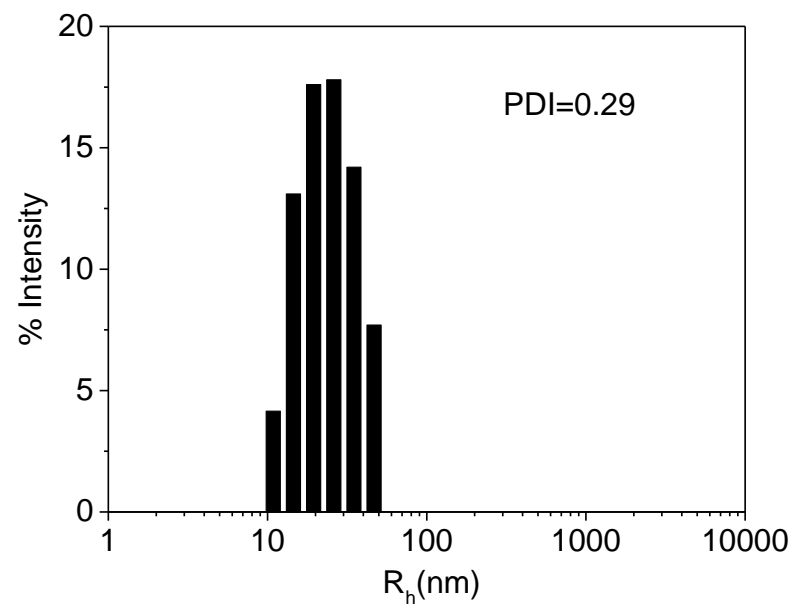

(b) 


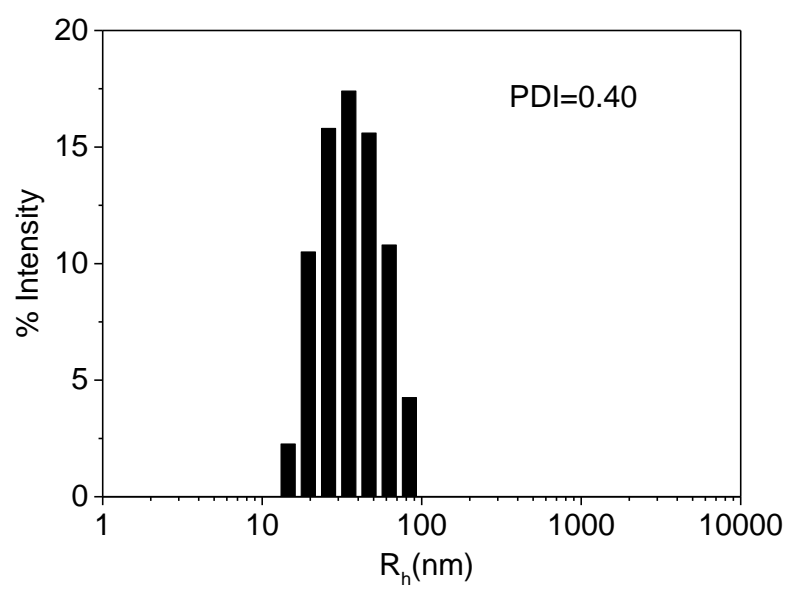

(c)

Figure S7. The mean hydrodynamic diameter distributions of $\mathrm{Fe}_{3} \mathrm{O}_{4} / \mathrm{PIAgCHI}$ particles at $\mathrm{pH}$ of 8 (a); $\mathrm{Fe}_{3} \mathrm{O}_{4} / \mathrm{PAAgCHI}$ at $\mathrm{pH} 10$ (b), and $\mathrm{pH} 8$ (c). 


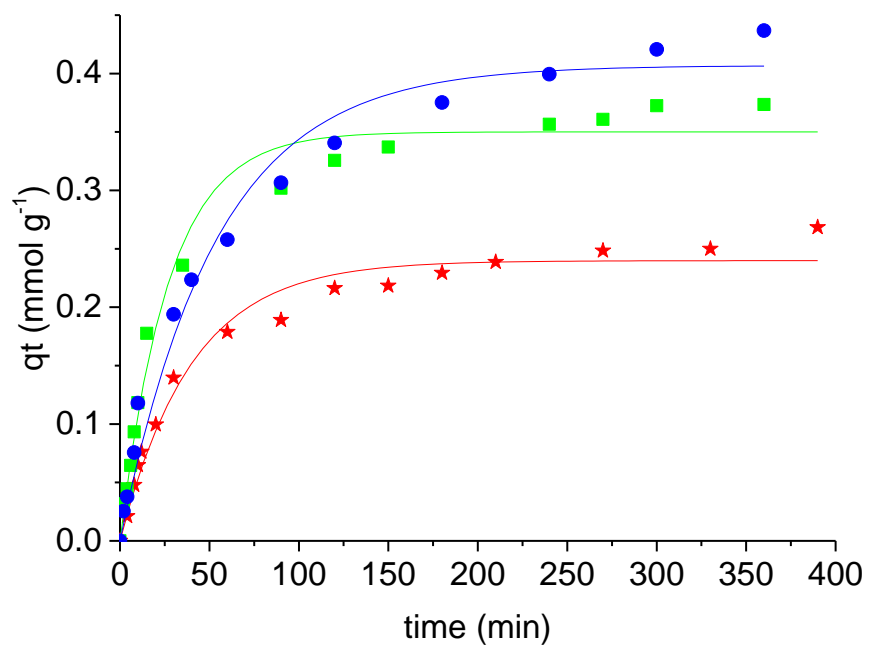

Figure S8. Adsorption kinetics of MB on PIAgCHI ( $\bullet$ ) $\mathrm{Fe}_{3} \mathrm{O}_{4} / \mathrm{PAAgCHI}(\square)$ and $\mathrm{Fe}_{3} \mathrm{O}_{4} / \mathrm{PIAgCHI}\left({ }^{*}\right) \mathrm{MNCs}$. The solid line is the pseudo-first-order (PSO) best-fit to the experiment data. 


\section{References}

(1) Hall-Edgefield, D. L.; Shi, T.; Nguyen, K.; Sidorenko, A. Hybrid Molecular Brushes with Chitosan Backbone: Facile Synthesis and Surface Grafting. ACS Appl Mater Inter 2014, 6, 22026-22033

(2) Wang, H. T.; Ma, H. Y.; Zheng, W.; An, D. D.; Na, C. Z. Multifunctional and Recollectable Carbon Nanotube Ponytails for Water Purification. ACS Appl Mater Inter 2014, 6, 9426-9434.

(3) Liu, F.; Chung, S.; Oh, G.; Seo, T. S. Three-Dimensional Graphene Oxide Nanostructure for Fast and Efficient Water-Soluble Dye Removal. ACS Appl Mater Inter 2012, 4, 922-927.

(4) Yu, L.; Liu, X. K.; Yuan, W. C.; Brown, L. J.; Wang, D. Y. Confined Flocculation of Ionic Pollutants by Poly(L-dopa)-Based Polyelectrolyte Complexes in Hydrogel Beads for Three-Dimensional, Quantitative, Efficient Water Decontamination. Langmuir 2015, 31, 6351-6366.

(5) Luo, W. J.; Gao, Q.; Wu, X. L.; Zhou, C. G. Removal of Cationic Dye (Methylene Blue) from Aqueous Solution by Humic Acid-Modified Expanded Perlite: Experiment and Theory. Sep Sci Technol 2014, 49, 2400-2411.

(6) Yao, Y. J.; Xu, F. F.; Chen, M.; Xu, Z. X.; Zhu, Z. W. Adsorption behavior of methylene blue on carbon nanotubes. Bioresource Technol 2010, 101, 3040-3046.

(7) Liu, T. H.; Li, Y. H.; Du, Q. J.; Sun, J. K.; Jiao, Y. Q.; Yang, G. M.; Wang, Z. H.; Xia, Y. Z.; Zhang, W.; Wang, K. L.; Zhu, H. W.; Wu, D. H. Adsorption of methylene blue from aqueous solution by graphene. Colloid Surface B 2012, 90, 197-203.

(8) Mohamed, M. H.; Dolatkhah, A.; Aboumourad, T.; Dehabadi, L.; Wilson, L. D. Investigation of templated and supported polyaniline adsorbent materials. RSC Adv 2015, 5, 6976-6984.

(9) Wang, L.; Wu, X. L.; Xu, W. H.; Huang, X. J.; Liu, J. H.; Xu, A. W. Stable Organic-Inorganic Hybrid of Polyaniline/alpha-Zirconium Phosphate for Efficient Removal of Organic Pollutants in Water Environment. ACS Appl Mater Inter 2012, 4, 2686-2692.

(10) Ma, J.; Yu, F.; Zhou, L.; Jin, L.; Yang, M. X.; Luan, J. S.; Tang, Y. H.; Fan, H. B.; Yuan, Z. W.; Chen, J. H. Enhanced Adsorptive Removal of Methyl Orange and Methylene Blue from Aqueous Solution by Alkali-Activated Multiwalled Carbon Nanotubes. ACS Appl Mater Inter 2012, 4, 5749-5760. 
(11) Xia, Y. X.; Dai, X. X.; Huang, S. T.; Tian, X. M.; Yang, H. Y.; Li, Y. C.; Liu, Y.; Zhao, M. J. Fast and highly efficient removal of methylene blue by a novel EDTAD-modified magnetic chitosan material. Desalin Water Treat 2013, 51, 7586-7595.

(12) Ghorai, S.; Sarkar, A.; Raoufi, M.; Panda, A. B.; Schonherr, H.; Pal, S. Enhanced Removal of Methylene Blue and Methyl Violet Dyes from Aqueous Solution Using a Nanocomposite of Hydrolyzed Polyacrylamide Grafted Xanthan Gum and Incorporated Nanosilica. ACS Appl Mater Inter 2014, 6, 4766-4777.

(13) Yan, B.; Chen, Z.; Cai, L.; Chen, Z.; Fu, J.; Xu, Q. Fabrication of polyaniline hydrogel: Synthesis, characterization and adsorption of methylene blue. Applied Surface Science 2015, 356, 39-47.

(14) Zhu, S.; Fang, S.; Huo, M.; Yu, Y.; Chen, Y.; Yang, X.; Geng, Z.; Wang, Y.; Bian, D.; Huo, H. A novel conversion of the groundwater treatment sludge to magnetic particles for the adsorption of methylene blue. Journal of Hazardous Materials 2015, 292, 173-179.

(15) Cho, D.-W.; Jeon, B.-H.; Chon, C.-M.; Schwartz, F. W.; Jeong, Y.; Song, H. Magnetic chitosan composite for adsorption of cationic and anionic dyes in aqueous solution. Journal of Industrial and Engineering Chemistry 2015, 28, 60-66. 